\title{
Physics of Waveguide Photodetectors with Integrated Amplification
}

\author{
J. Piprek, D. Lasaosa, D. Pasquariello, and J. E. Bowers \\ Electrical and Computer Engineering Department \\ University of California, Santa Barbara, CA 93106
}

\begin{abstract}
The promising concept of waveguide photodetection with integrated amplification is evaluated by self-consistent device simulation. Such integrated amplification detectors have the potential to achieve simultaneously high saturation power, high speed, high responsivity, and quantum efficiencies well above one. Our example design vertically combines a bulk photodetector ridge region with laterally confined quantum wells for amplification. The current flow in the three-terminal device exhibits ground current reversal with increasing light power. The net optical gain is evaluated for different waveguide modes. For the dominating mode, the detector responsivity is shown to scale with the device length, reaching quantum efficiencies larger than $100 \%$.
\end{abstract}

Keywords: photodetector, waveguide, amplifier, integrated photonics, optical gain, III-V semiconductor devices, numerical simulation

\section{INTRODUCTION}

In waveguide photodetectors, photon flux and carrier flux are perpendicular to each other, which enables highdata-rate applications since thin absorption layers allow for short transit times of the generated carriers. The detection efficiency is increased by using long waveguides. Ideally, every photon should generate one electronhole pair giving $100 \%$ quantum efficiency. However, only a fraction of the optical signal is usually converted into an electrical signal. To obtain stronger electrical output power, the optical input power may be increased by preamplification. This method is limited by saturation effects, as intense light leads to a large carrier density which changes the behaviour of the detector. A solution to this problem was recently proposed by integrating detector and amplifier. ${ }^{1}$

Our traveling-wave amplification photodetector (TAP detector) simultaneously amplifies and absorbs the incoming light. The schematic cross section a vertically coupled device is shown in Fig. 1. The top GaAs ridge is the detecting layer, all other semiconductor layers exhibit a larger bandgap. Light amplification is provided by multiple GaAs quantum wells. The amplifier $p n$ diode is operated at forward bias whereas the detector diode is reverse biased. Electrical and optical confinement in the center of the device is provided by the ridge-waveguide structure as well as by lateral oxidation. The continuous amplification of the optical wave makes it possible to maintain a constant optical power along the waveguide despite the absorption. With long devices, much higher output power than in conventional photodetectors can be achieved. Details of the device design are evaluated in the following using advanced two-dimensional (2D) device simulation software. ${ }^{2}$

\section{DEVICE STRUCTURE AND MATERIAL PROPERTIES}

The layer structure of the device is listed in Tab. 1. Refractive index data for AlGaAs are obtained from Ref. ${ }^{3}$ Oxidation layers exhibit a high $\mathrm{Al}$ mole fraction which lowers the refractive index. The vertical index profile is plotted in Fig. 2 together with several vertical waveguide modes. The behavior of these optical modes will be analyzed in the next Section. Lateral mode confinement is provided by the top ridge as well as by the oxide layers. A value of 1.7 is used for the refractive index of aluminum oxide. For the device investigated here, the width of the oxide aperture is equal to the ridge width of $2 \mu \mathrm{m}$.

Email: piprek@ieee.org, Telephone: 1-805-893-4051, Fax: 1-805-893-5440 


\begin{tabular}{|c|c|c|c|c|}
\hline Parameter & $l$ & $N_{d o p}$ & $\mu$ & $n_{r}$ \\
\hline Unit & $\mathrm{nm}$ & $1 / \mathrm{cm}^{3}$ & $\mathrm{~cm}^{2} / \mathrm{Vs}$ & - \\
\hline i-GaAs (absorption) & 300 & $1 \times 10^{16}$ & 290 & $\overline{3.650}$ \\
\hline $\mathrm{n}-\mathrm{Al}_{0.15} \mathrm{Ga}_{0.85} \mathrm{As}$ (cladding) & 100 & $5 \times 10^{18}$ & 1000 & 3.513 \\
\hline $\mathrm{n}-\mathrm{Al}_{0.2} \mathrm{Ga}_{0.8} \mathrm{As}$ (cladding) & 300 & $1 \times 10^{18}$ & 1300 & 3.482 \\
\hline $\mathrm{n}-\mathrm{Al}_{x} \mathrm{Ga}_{1-x}$ As (grading $\mathrm{x}=0.9$ to 0.2 ) & 16.5 & $1 \times 10^{18}$ & graded & graded \\
\hline $\mathrm{n}-\mathrm{Al}_{0.9} \mathrm{Ga}_{0.1} \mathrm{As}$ (oxidation) & 6 & $1 \times 10^{18}$ & 60 & 3.046 \\
\hline $\mathrm{n}-\mathrm{Al}_{0.98} \mathrm{Ga}_{0.02} \mathrm{As}$ (oxidation) & 32 & $1 \times 10^{18}$ & 65 & 2.996 \\
\hline $\mathrm{n}-\mathrm{Al}_{0.9} \mathrm{Ga}_{0.1} \mathrm{As}$ (oxidation) & 6 & $1 \times 10^{18}$ & 60 & 3.046 \\
\hline $\mathrm{n}-\mathrm{Al}_{x} \mathrm{Ga}_{1-x} \mathrm{As}$ (grading $\mathrm{x}=0.15$ to 0.9 ) & 18.2 & $1 \times 10^{18}$ & graded & graded \\
\hline $\mathrm{n}-\mathrm{Al}_{0.15} \mathrm{Ga}_{0.85} \mathrm{As}$ (waveguide) & 90 & $1 \times 10^{18}$ & 1800 & 3.513 \\
\hline $\mathrm{i}-\mathrm{Al}_{0.15} \mathrm{Ga}_{0.85} \mathrm{As}$ (waveguide) & 10 & $1 \times 10^{16}$ & 150 & 3.513 \\
\hline i-GaAs (quantum well) & 8 & $1 \times 10^{16}$ & 310 & 3.650 \\
\hline i- $\mathrm{Al}_{0.15} \mathrm{Ga}_{0.85} \mathrm{As}$ (barrier) & 8 & $1 \times 10^{16}$ & 260 & 3.513 \\
\hline i-GaAs (quantum well) & 8 & $1 \times 10^{16}$ & 310 & 3.650 \\
\hline $\mathrm{i}-\mathrm{Al}_{0.15} \mathrm{Ga}_{0.85} \mathrm{As}$ (barrier) & 8 & $1 \times 10^{16}$ & 260 & 3.513 \\
\hline i-GaAs (quantum well) & 8 & $1 \times 10^{16}$ & 310 & 3.650 \\
\hline i- $\mathrm{Al}_{0.15} \mathrm{Ga}_{0.85} \mathrm{As}$ (barrier) & 8 & $1 \times 10^{16}$ & 260 & 3.513 \\
\hline i-GaAs (quantum well) & 8 & $1 \times 10^{16}$ & 310 & 3.650 \\
\hline i- $\mathrm{Al}_{0.15} \mathrm{Ga}_{0.85} \mathrm{As}$ (waveguide) & 68 & $1 \times 10^{16}$ & 260 & 3.513 \\
\hline p- $\mathrm{Al}_{0.2} \mathrm{Ga}_{0.8} \mathrm{As}$ (waveguide) & 20 & $5 \times 10^{17}$ & 135 & 3.482 \\
\hline p- $\mathrm{Al}_{x} \mathrm{Ga}_{1-x}$ As (grading $\mathrm{x}=0.9$ to 0.2 ) & 18.2 & $1 \times 10^{18}$ & graded & graded \\
\hline $\mathrm{p}-\mathrm{Al}_{0.9} \mathrm{Ga}_{0.1} \mathrm{As}$ (oxidation) & 6 & $3 \times 10^{17}$ & 60 & 3.046 \\
\hline $\mathrm{p}-\mathrm{Al}_{0.98} \mathrm{Ga}_{0.02} \mathrm{As}$ (oxidation) & 32 & $3 \times 10^{17}$ & 60 & 2.996 \\
\hline p- $\mathrm{Al}_{0.9} \mathrm{Ga}_{0.1} \mathrm{As}$ (oxidation) & 6 & $3 \times 10^{17}$ & 60 & 3.046 \\
\hline $\mathrm{p}-\mathrm{Al}_{x} \mathrm{Ga}_{1-x} \mathrm{As}$ (grading $\mathrm{x}=0.2$ to 0.9 ) & 16.5 & $2 \times 10^{18}$ & graded & graded \\
\hline $\mathrm{p}-\mathrm{Al}_{0.2} \mathrm{Ga}_{0.8} \mathrm{As}$ (cladding) & 300 & $5 \times 10^{17}$ & 135 & 3.482 \\
\hline $\mathrm{p}-\mathrm{Al}_{0.2} \mathrm{Ga}_{0.8} \mathrm{As}$ (cladding) & 200 & $2 \times 10^{18}$ & 100 & 3.482 \\
\hline $\mathrm{p}-\mathrm{Al}_{0.2} \mathrm{Ga}_{0.8} \mathrm{As}$ (cladding) & 100 & $5 \times 10^{18}$ & 75 & 3.482 \\
\hline $\mathrm{i}-\mathrm{Al}_{0.5} \mathrm{Ga}_{0.5} \mathrm{As}$ (sublayer) & 3000 & $1 \times 10^{16}$ & 160 & 3.295 \\
\hline
\end{tabular}

Table 1. Epitaxial layer structure of the TAP detector ( $l$ - layer thickness, $N_{d o p}$ - doping, $\mu$ - majority carrier mobility, $n_{r}$ - refractive index, room temperature). Intrinsic (i) layers are assumed to exhibit low p-type background doping. 


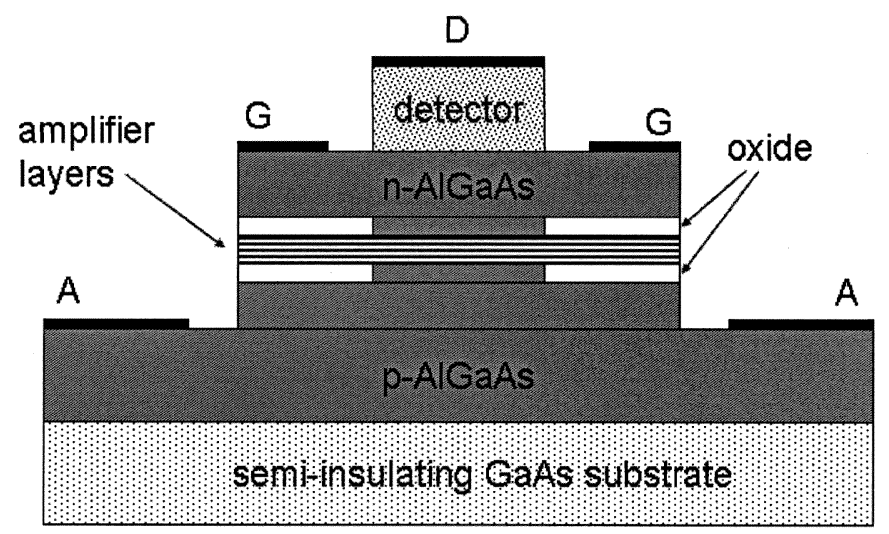

Figure 1. Cross section of the TAP detector (D - detector contact, G - ground contact, A - amplifier contact).

The $\mathrm{Al}_{x} \mathrm{Ga}_{1-x}$ As system converts from a direct semiconductor for $x<0.45$ to an indirect semiconductor for $x>0.45$. Our device contains both types of materials. The hole transport is hardly affected by this transition. However, most electrons entering the n-doped oxidation layers are transferred from the $\Gamma$ valley into the $X$ side valley of the conduction band. Due to the high $\mathrm{Al}$ mole fraction, a negligible number of electrons is still traveling in the $\Gamma$ valley. Thus, the lower band edge is used in calculating the carrier transport across heterointerfaces. The common band offset ratio of $\Delta E_{c} / \Delta E_{v}=60: 40$ is employed at all interfaces. Fig. 3 shows the band diagram at the vertical axis of the device. Due to the grading and doping profile used, the valence band edge of the oxidation layers is almost flat on the p-doped side and it hardly affects the hole injection into the multi-quantum well (MQW) amplification region. The GaAs detector region is reverse biased.

The conduction bands of the active GaAs layers are assumed parabolic and the non-parabolic valence bands are calculated by the two-band $\vec{k} \cdot \overrightarrow{\mathbf{p}}$ method. ${ }^{4}$ The free carrier model for stimulated band-to-band transitions is employed to compute both gain and absorption for the amplification layers and the detection layer, respectively. ${ }^{5}$ The calculated spectra are plotted in Fig. 4 for different carrier densities. At low carrier density (reverse bias), the bulk GaAs layer exhibits an absorption coefficient of about $10^{4} \mathrm{~cm}^{-1}$ at $840 \mathrm{~nm}$ wavelength which was adjusted to fit experimental data. With forward bias, the carrier density in the QW amplification layers is much higher. At $840 \mathrm{~nm}$ wavelength, the gain reaches $6000 \mathrm{~cm}^{-1}$ for a carrier density of $8 \times 10^{18} \mathrm{~cm}^{-3}$. However, the total amplification layer thickness of $32 \mathrm{~nm}$ is much smaller than the absorption layer thickness of $300 \mathrm{~nm}$. Thus, optical waveguide modes are preferred that exhibit little overlap with the detector region.

\section{WAVEGUIDE MODE ANALYSIS}

Three main vertical modes of our device are shown in Fig. 2 and mode (c) is given as 2D contour plot in Fig. 5. Mode (d) is mainly located in the detector region, mode (a) in the amplifier region and mode (c) does not show much overlap with any active layer. The optical confinement factors of these three modes are given in Tab. 2 for both the active regions. The total modal gain of each mode can be estimated as

$$
g_{\text {mode }}=\Gamma_{a m p} g_{a m p}+\Gamma_{d e t} g_{d e t}
$$

with $g_{a m p}$ and $g_{d e t}$ from Fig. 4. The normalized optical power in travel direction is given in Fig. 6 for all three modes. The detector mode (d) is completely absorbed after a short travel distance. The amplifier mode (a) also 


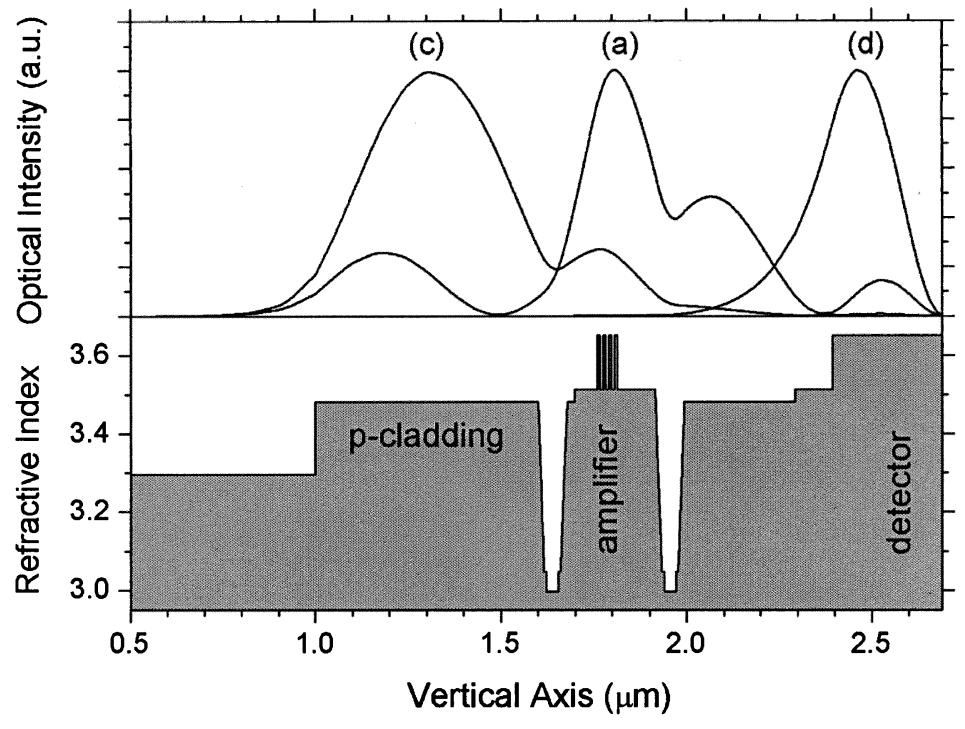

Figure 2. Vertical profile for different waveguide modes (top) and for the refractive index at $840 \mathrm{~nm}$ wavelength (bottom).

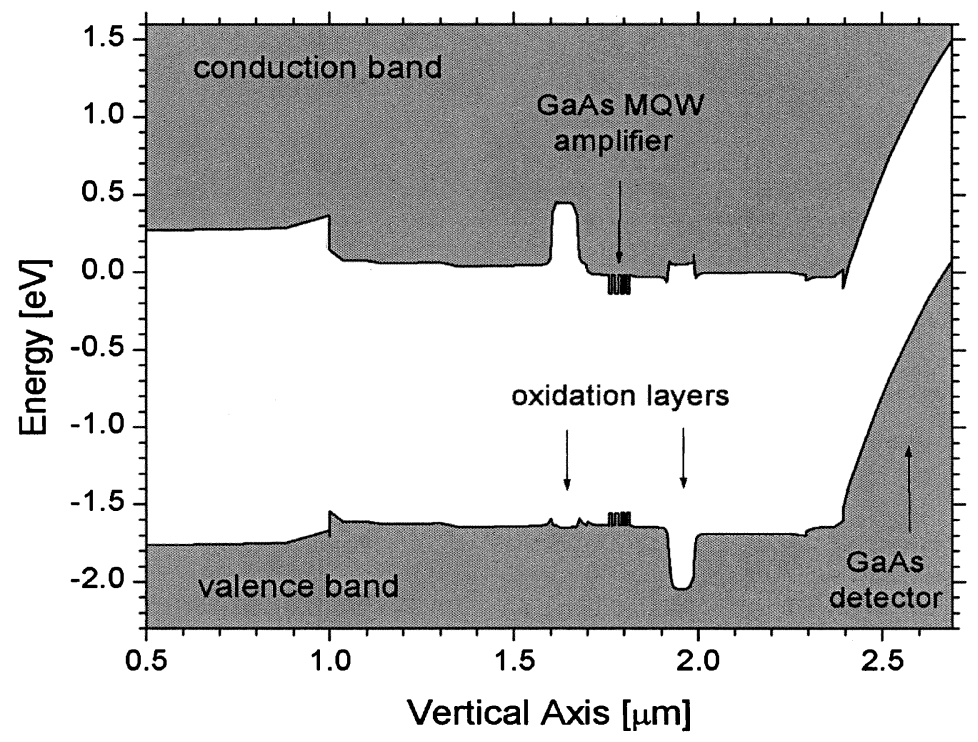

Figure 3. Band diagram at the vertical axis with $V_{D}=-1 \mathrm{~V}$ detector voltage and $I_{A}=20 \mathrm{~mA}$ amplifier current.

\begin{tabular}{|l|l|l|}
\hline Mode & $\Gamma_{a m p}$ & $\Gamma_{\text {det }}$ \\
\hline$(\mathrm{d})$ & 0.00014 & 0.6769 \\
\hline$(\mathrm{c})$ & 0.0165 & 0.0028 \\
\hline$(\mathrm{a})$ & 0.0684 & 0.0524 \\
\hline
\end{tabular}

Table 2. Optical confinement factors $\Gamma_{a m p}$ and $\Gamma_{d e t}$ of the vertical modes in Fig. 2 for amplification and detection layers, respectively. 


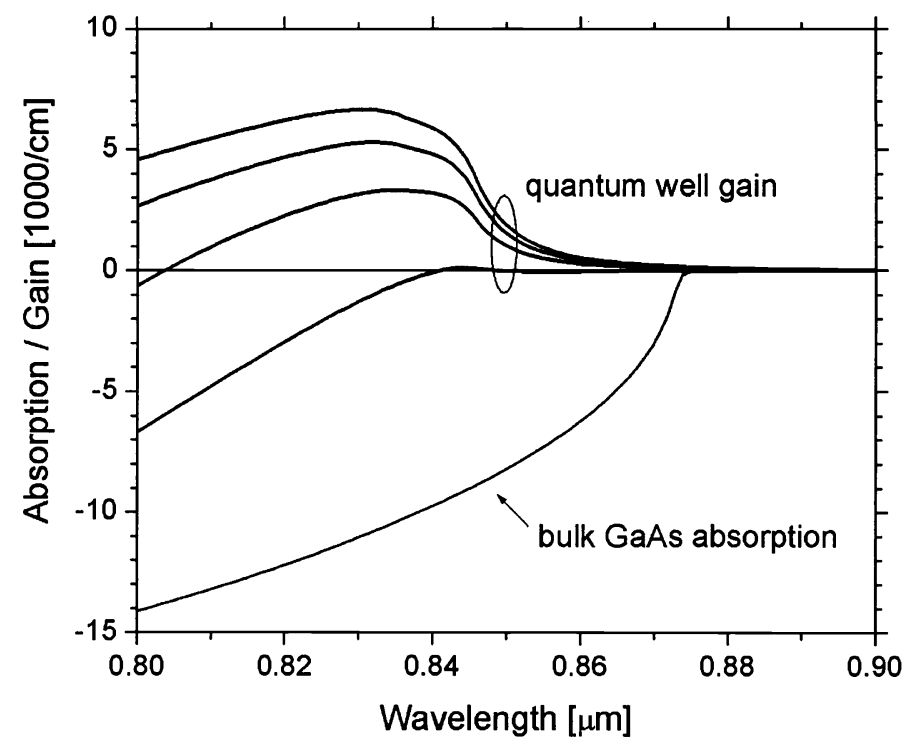

Figure 4. Gain and absorption spectra for quantum well and ridge, respectively. Gain spectra are calculated at four carrier densities: $2,4,6$, and $8 \times 10^{18} \mathrm{~cm}^{-3}$, the absorption is calculated at $10^{16} \mathrm{~cm}^{-3}$.

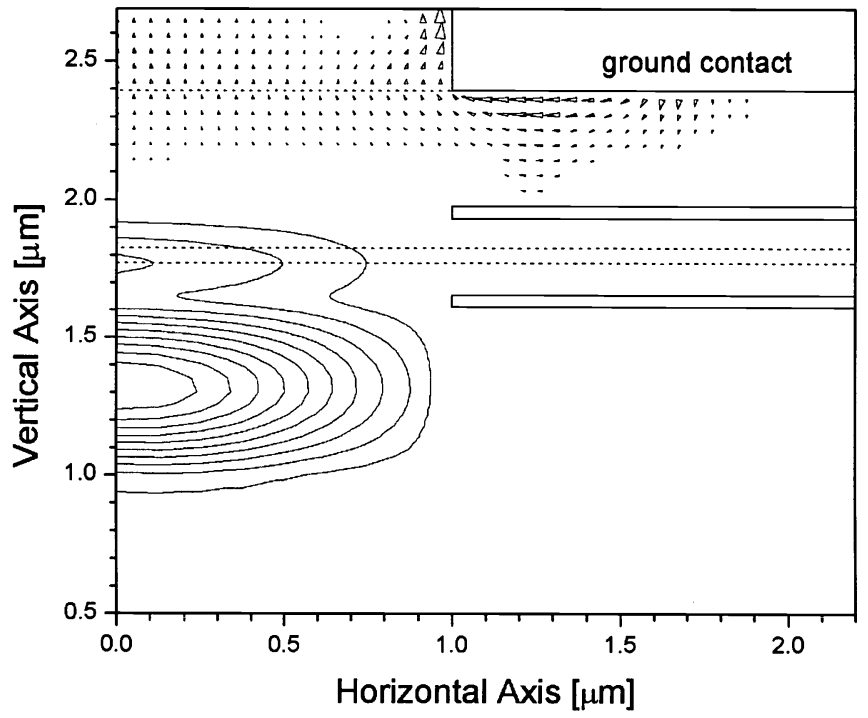

Figure 5. Contours: 2D intensity distribution of mode (c). Arrows: current density at $I_{A}=20 \mathrm{~mA}$ amplifier current, $V_{D}=-1 \mathrm{~V}$ detector voltage, and $P_{2}=100 \mathrm{~mW}$ modal power (the arrows scale with the current density and disappear for less than $10 \%$ of the maximum). 


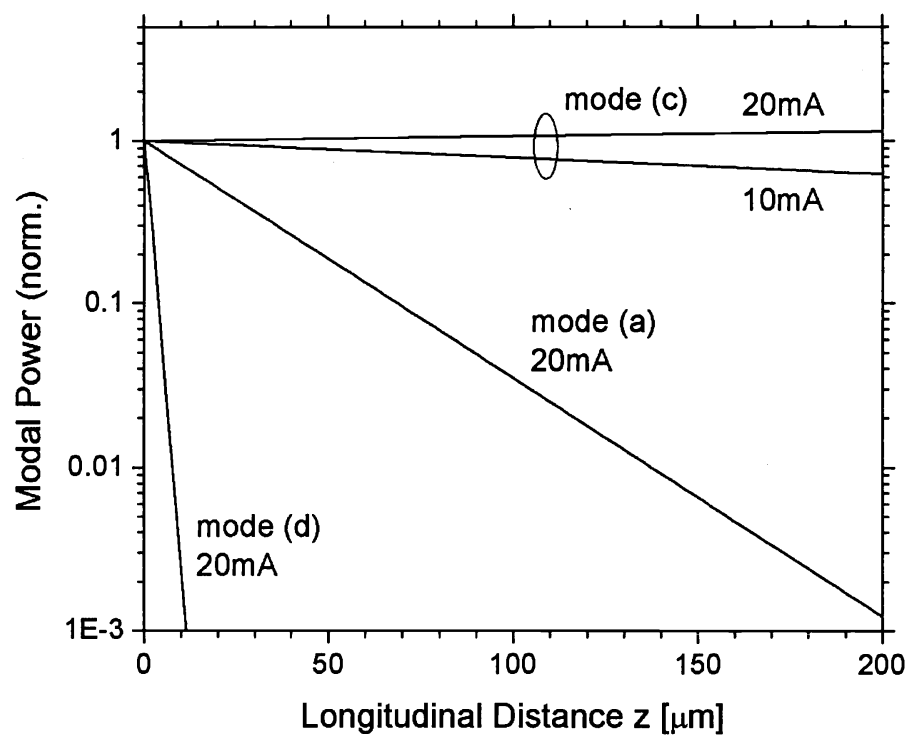

Figure 6. Normalized modal power in travel direction at $840 \mathrm{~nm}$ wavelength for all 3 modes with the amplifier current given as parameter.

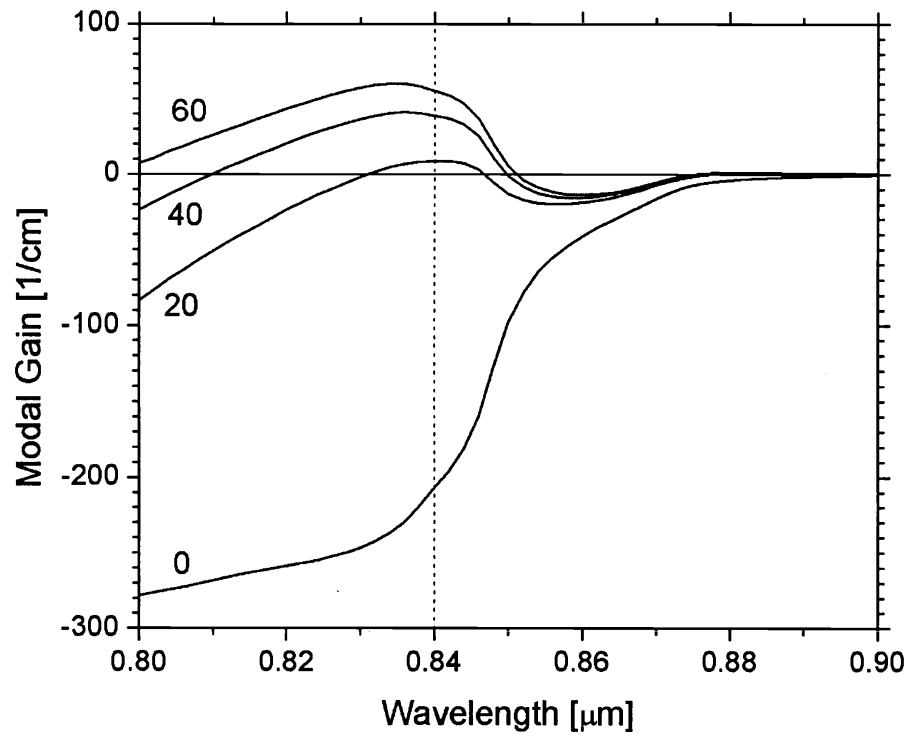

Figure 7. Modal gain spectrum for mode (c) from Fig. 2 at different amplifier currents [mA].

suffers from net absorption. Only the cladding mode (c) exhibits positive gain with sufficient pumping. Its net modal gain spectrum is plotted in Fig. 7 as function of the amplifier current. At the optimum wavelength of $840 \mathrm{~nm}$, mode (c) shows zero net gain for $17 \mathrm{~mA}$ amplifier current, which gives constant optical power along the waveguide. The other two modes do not reach zero net gain for any reasonable pumping current.

Many different waveguide modes may be excited by coupling light into the device, e.g., from an optical fiber. 


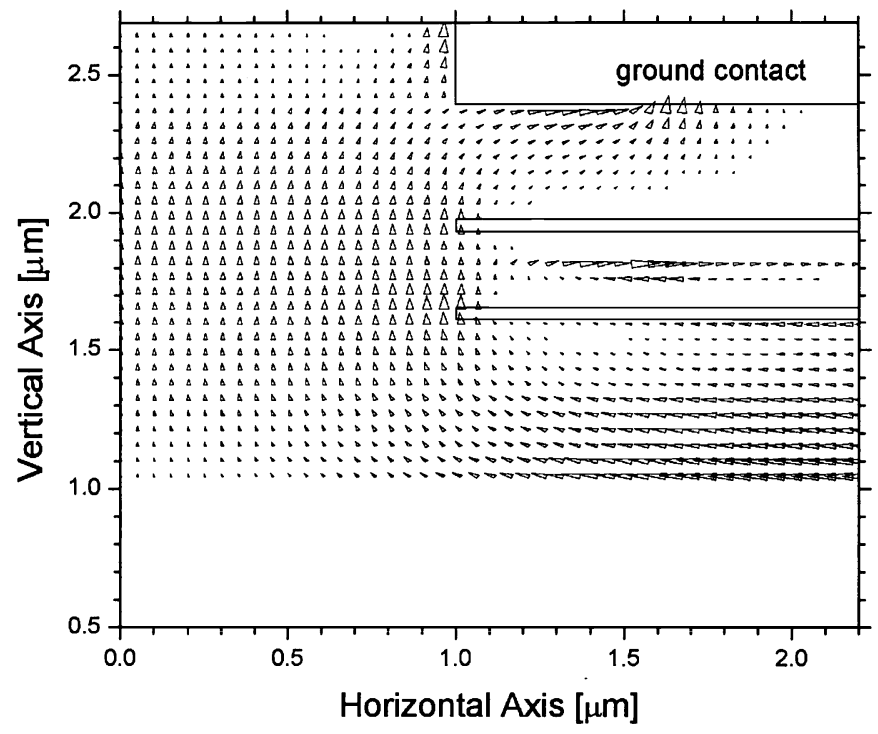

Figure 8. Vector plot of the current density at $I_{A}=17 \mathrm{~mA}$ amplifier current and $P_{2}=10 \mathrm{~mW}$ modal power (the size of the arrows scales with the current density).

Most of these modes are quickly absorbed by the thick detector region. Only those modes are useful in our device for which about the same number of photons is generated in the quantum wells and absorbed in the detector. This transfer of photons is the basic idea of the TAP detector. Photons that are multiplied by stimulated emission stay within the same mode. Thus, in our device example, the incoming signal needs to be mainly coupled into modes that are guided by the lower cladding layers.

\section{DETECTOR RESPONSIVITY}

In the following, we investigate the detector's response to the cladding mode (c) for the special case of constant longitudinal mode power $\left(I_{A}=17 \mathrm{~mA}\right)$. Figure 8 shows a vector plot of the current density distribution at low optical power $\left(P_{2}=10 \mathrm{~mW}, V_{D}=-1 \mathrm{~V}\right)$. The plot reveals current crowding at the edges of the oxide aperture as well as lateral current leakage within the MQW amplifier region. The detector generates about $I_{D} \approx 5 \mathrm{~mA}$ current, which is still smaller than $I_{A}$ so that the amplifier dominates the direction of the current flow at the ground contact. This situation changes with higher modal power. At $P_{2}=100 \mathrm{~mW}$, the detector current is close to $50 \mathrm{~mA}$ and the current flow at the ground electrode reverses (cf. Fig. 5).

Figure 9 plots the photocurrent as function of the modal power for two different detector lengths $L$. Due to its little overlap with the absorption layer, mode (c) shows a very linear slope without any detector saturation effect up to $100 \mathrm{~mW}$ input power. The detector responsivity is $d I_{D} / d P=0.53 \mathrm{~A} / \mathrm{W}$ at $L=200 \mu \mathrm{m}$ and it is twice as high with double the device length. The differential quantum efficiency is given by

$$
\eta_{\text {det }}=\frac{d I_{D}}{d P} \frac{\hbar \omega}{q}
$$

and it surpasses $100 \%$ for $L>257 \mu \mathrm{m}$ in our case. With a $400 \mu \mathrm{m}$ long detector, each incident photon generates on average 1.55 electrons due to photon multiplication in the gain region. 


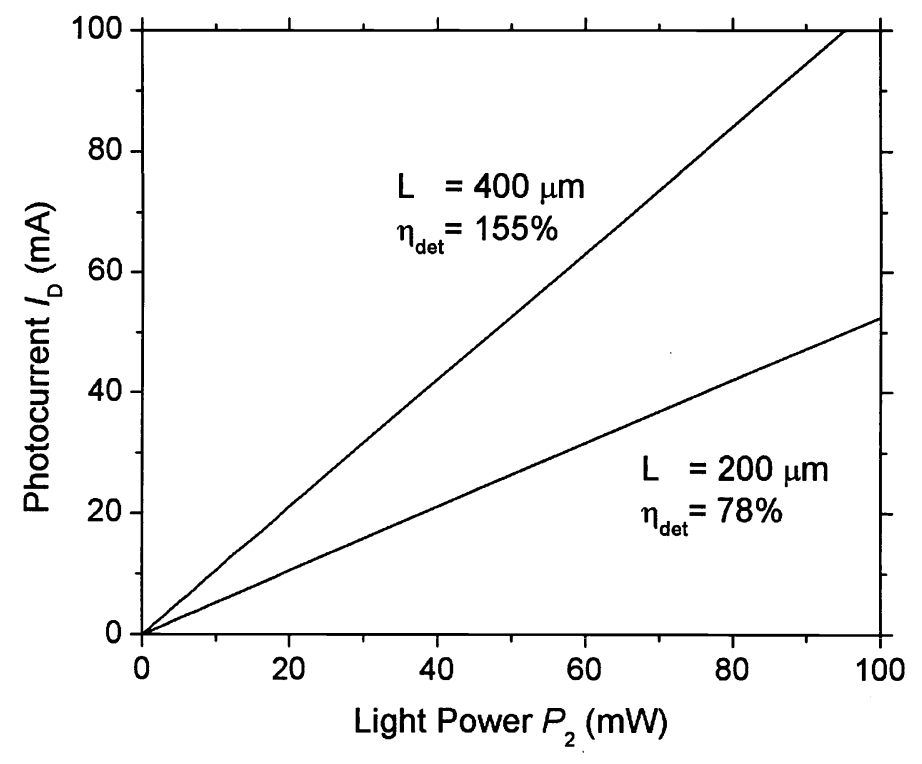

Figure 9. Photocurrent as function of second mode power at $840 \mathrm{~nm}$ wavelength with $I_{A}=17 \mathrm{~mA}$ pump current and $V_{D}=-1 \mathrm{~V}$ detector voltage. The quantum efficiency $\eta_{\text {det }}$ scales with the detector length $L$ since the modal power is constant in longitudinal direction.

\section{SUMMARY}

The concept of waveguide photodetection with integrated amplification is evaluated by two-dimensional device simulation. A bulk GaAs photodetector region is combined with GaAs quantum wells for amplification. The current flow in the three-terminal device is analyzed. The net optical gain is calculated for different waveguide modes, identifying the preferred mode of operation. For this mode, the detector responsivity is shown to scale with the device length, reaching a quantum efficiency larger than $100 \%$.

\section{REFERENCES}

1. D. Lasaosa, Y. J. Chiu, J. Piprek, and J. E. Bowers, "Traveling-wave amplification photodetector (TAP detector)," in 13th Lasers and Electro-Optics Society Annual Meeting, (Piscataway), pp. 260-261, Institute of Electrical and Electronic Engineers, 2000.

2. APSYS by Crosslight Software, 2001.

3. M. A. Afromowitz, "Refractive index of GaAlAs," Solid State Comm., vol. 15, pp. 59-63, 1974.

4. S. L. Chuang, Physics of Optoelectronic Devices. New York: Wiley, 1995.

5. J. Piprek, Semiconductor Optoelectronic Devices - Introduction to Physics and Simulation. San Diego: Academic Press, 2003. 